\title{
Clinical Spectrum of Obsessive-Compulsive Disorder in Patients From Central India
}

\section{Ragini Patil', Tushar Patil', Vishal Kumar Shah ${ }^{3}$}

Section: Healthcare

Sci. Journal Impact

Factor: 6.1 (2018)

ICV: 90.90 (2018)

Scopus'

\begin{abstract}
Assistant Professor Department of Psychiatry, Datta Meghe Medical College, Shalinitai Meghe Hospital and Research Centre, Nagpur-44n10; ${ }^{2}$ Assistant Professor, Department of General Medicine, Datta Meghe Medical College, Shalinitai Meghe Hospital and Research Centre, Nagpur-441110; ${ }^{3}$ Assistant Professor Dept. of Psychiatry, Jawaharlal Nehru Medical College, Datta Meghe lnstitute of Medical Sciences, Sawangi, Wardha-442001.
\end{abstract}

\section{ABSTRACT}

Introduction: Obsessive-Compulsive Disorder (OCD) is an important cause of psychiatric morbidity in India. It is often coexistent with other psychiatric disorders such as Schizophrenia. There is no study describing OCD clinical spectrum from Central India.

Aims \& Objectives: To determine the clinical spectrum of obsessive-compulsive symptoms/disorder, to study the type of obsessive-compulsive symptoms/disorder, and to evaluate demographic \& phenomenological variables.

Material \& Methods: This was a prospective observational study in a tertiary teaching hospital. All patients presenting to the outpatient clinics or admitted to the ward were evaluated for symptoms of obsessive-compulsive disorder. All these patients who were drug naïve were selected after fulfilling the inclusion and exclusion criteria. The patients were enrolled after obtaining written informed consent. The severity of OCD was assessed on YBOCS and global severity and improvement was assessed using the CGI scale at 12 weeks follow-up.

Results: We evaluated 105 patients, out of whom 26 patients had OCD/S. Thus the prevalence of OCD/S in our study was $24.7 \%$. Patients were in the age range of $19-58$ years, with nearly $51.4 \%$ patients being in the age range of $19-29$ years, $21.9 \%$ in the age range of 30-39 years, and 40-49 years respectively and with only $4.7 \%$ being more than 50 years. Overall male: female ratio was $1.5: 1$. It was seen that $50-60 \%$ of the patients had achieved some form of education and nearly more than $50 \%$ patients had achieved a secondary and higher levels of education. The mean duration of symptoms was 33.69 months. Predominantly obsessional thoughts and ruminations were in 04 (15.4\%); predominantly compulsive acts in 03 (11.5\%); Mixed obsessional thoughts and acts in 19 (73.1\%); Other obsessive-compulsive disorders in 0 (0\%); Obsessive-compulsive disorder, unspecified in 0 (0\%). Mixed OCD was seen in 19 (73.0\%); predominantly obsessional in $4(15.3 \%)$ and predominantly compulsive in $3(11.5 \%)$ patients. Types of obsessions were: Aggressive Obsessions in 0 (0\%); Contamination Obsessions in 14 (53.8\%); Sexual Obsessions in 04 (15.4\%); Hoarding / Saving Obsessions in 01 (3.8\%); Religious Obsessions in 03 (11.5\%); Obsessions with Need for Symmetry or Exactness in 01 (3.8\%); Somatic Obsessions in 01 (3.8\%) and Miscellaneous Obsessions in 02 (7.7\%) cases. Types of compulsions were: Cleaning/Washing in 14 (53.8\%); Checking in 04 (15.4\%); Repeating in 04 (15.4\%); Counting in 02 (7.7\%); Ordering/ Arranging in 01 (3.8\%); Hoarding/ Collecting in 0 (0\%) and Miscellaneous compulsions were seen in 01 (3.8\%) patients. At the end of 3 months then a statistically significant difference was noted on the YBOCS obsession score $(t=12.359, p<0.001)$, YBOCS compulsion score $(t=9.29, p<0.001)$, and total YBOCS score $(t=24.154, p<0.001)$ from the baseline scores.

Conclusions: We concluded that the prevalence of OCD in the population evaluated was $24.7 \%$. It affected young individuals with lower socioeconomic status. Majority of patients in Group A did not work and were unemployed. The majority of patients in both the groups belonged to lower socioeconomic strata. The majority of patients had mixed obsessional thought and compulsive acts pattern of OCD, followed by obsessional thoughts predominantly compulsive acts. The most common obsessions were obsessions of contamination, sexual obsession, religious obsession, savings, need for symmetry. At the end of 3 months, there was a marked reduction in obsessive-compulsive symptoms on YBOCS.

Key Words: OCD, YBOCS, Obsessive-compulsive, ECA

\section{INTRODUCTION}

The obsessive-compulsive (OC) disease has been extensively described and debated over the years. In the early years, it was believed that these affective and anxiety symptoms are a reactive manifestation of the perceived external or internal stressors ${ }^{1}$ People who display significant OC symptoms are commonly seen in clinical practice, and several groups have

\section{Corresponding Author:}

Dr. Tushar Patil, Assistant Professor Department of General Medicine, Datta Meghe Medical College, Shalinitai Meghe Hospital and Research Centre, Nagpur-441110; Email.id. ragini.tushar@gmail.com

ISSN: 2231-2196 (Print)

Received: 28.05 .2020
ISSN: 0975-5241 (Online)

Revised: 21.06 .2020
Accepted: 03.07.2020
Published: 21.07 .2020 
investigated OC symptoms. The National Institute of Mental Health Epidemiological Catchment Area (ECA) study that used the diagnostic interview schedule (DIS) to examine comorbidity in mental disorders reported a prevalence rate of co-occurring OCD and schizophrenia of $12.2 \%{ }^{2}$. Other investigators have reported greater symptom severity, suicide risk, and functional impairment, as well as the worse long-term outcome, in $\mathrm{OC}$ schizophrenia compared with non-OC schizophrenia ${ }^{3,4,5}$ A number of recent treatment studies and anecdotal case reports have shown an adjunctive selective serotonin reuptake inhibitor (SSRI) may be an effective intervention. The neurobiological implications of OC a phenomenon still remains obscure and there has been little progress in our understanding of the clinical and neurobiological significance of OC phenomenon. Further understanding of the OC phenomena may thus enlighten our endeavor to construct a more clinically meaningful and neurobiologically relevant subtyping strategy.

Hence, our study was planned to evaluate the clinical spectrum of OCD. The aims and objectives of this study were to determine the clinical spectrum of obsessive-compulsive symptoms/disorder, to study type of obsessive-compulsive symptoms/disorder, and to evaluate demographic and phenomenological variables.

\section{MATERIAL AND METHODS}

The study was approved by the Institutional Ethics committee of the parent institute. All participants were informed about the study protocol, clinical evaluation, and the treatments involved in the study. Written informed consent was obtained from all participants. The study was performed in accordance with the provisions of the Declaration of Helsinki.

Study design: Prospective observational study.

Place of study: Cases were enrolled from the patients attending outpatient clinic and patients admitted in the Department of Psychiatry, Datta Meghe Medical College, Shalinitai Meghe Hospital and Research Centre in collaboration with Jawaharlal Nehru Medical College, Datta Meghe Institute of Medical Sciences, Sawangi, Wardha, Maharashtra.

Study population: All patients presenting to the outpatient clinic or admitted to the ward were evaluated for symptoms of obsessive-compulsive disorder. All these patients who were drug naïve were selected after fulfilling the inclusion and exclusion criteria. The patients were enrolled after obtaining written informed consent.

\section{Duration of study:}

Patient enrollment, data collection, and follow-up were done over a period of 12 months, and data analysis was done over the next 6 months. Thus the total duration of the study was 18 months. This was a prospective observational study that also evaluated the response to treatment with medications and follow up was done 12 weeks after initiation of treatment in each patient.

All the patients who were diagnosed as OCD and those who were drug naïve, were subjected to following inclusion criteria:

\section{Inclusion criteria:}

1. Age group: Above 18 years

2. Clinically diagnosed cases of OCD as per ICD10 criteria

3. No previous exposure to psychiatric medications (drug naive)

4. Informed consent of patients

The exclusion criteria were as follows:

\section{Exclusion Criteria:}

1. Patients having other medical comorbidities.

2. Patients already diagnosed as having psychiatric illness and on medications.

3. Already diagnosed cases of OCD on treatment.

Methodology: Written informed consent to participate in the study was taken from the patient's relatives, prior to enrollment. Complete history taking and thorough clinical evaluation were done in each patient as per protocol. The clinical features of the study patients further carefully analyzed for the presence of OCD as per the ICD 10 criteria for obsessivecompulsive disorder. A semi-structured proforma was used to collect information on the various demographic variables, phenomenological factors duration of OCD/OCS, number of hospitalizations for the same, and scales pertaining to the aims of the study. The severity of OCD was assessed on YBOCS and global severity and improvement were assessed using the CGI scale.

\section{Yale Brown obsessive compulsive symptom scale:}

It is a clinician-administered symptom severity rating scale for $\mathrm{OCD}^{6}$. Before rating on the scale, the target symptoms' are identified by applying the YBOCS symptoms checklist. YBOCS is a 10 item scale that consists of five rating dimensions of Obsessions and Compulsions each. The items are each scored on four-point scale from $0=$ "no symptom" to 4 = "extreme symptom". The sum of the first five items ranges from 0 to 20 is a severity index for obsessions and the sum of the last five ranging from 0 to 20 is a severity index for compulsions. The translation of total score ranging from 0 to 40 gives the index of overall severity.

\section{Clinical global impression scale:}


It is a brief clinician-rated scale that measures the illness severity, global improvement, and therapeutic response ${ }^{7}$. The CGI severity scale score is rated on 7 point scale for illness severity that ranges from 1-normal to 7 amongst the most severely ill patients. The CGI global improvement score ranges from1-very much improved to very much worse. The therapeutic response takes in to account both therapeutic effect and treatment-related complications. In the CGI scale each component is rated separately and doesn't yield a global score.

\section{Outcome Measures}

The outcome of patients was assessed clinically with CGI scores at 12 weeks for all patients.

\section{Statistical analysis}

All categorical variables were expressed as numbers and percentages. Continuous variables were expressed as mean \pm standard deviation. Comparison of categorical variables between the two groups was done with Pearson's chi-square test. Fisher's exact test was used for small numbers. Continuous variables were compared between the two groups with the student's t-test for two independent samples. Mann Whitney $\mathrm{U}$ test was used for nonparametric data. Continuous variables at baseline and three months follow up were compared using the paired t-test for related samples.

\section{RESULTS}

Patients were evaluated for the presence of obsessive-compulsive disorder/symptoms. The severity of $\mathrm{OCD} / \mathrm{S}$ was studied by the Yale-Brown Obsessive-Compulsive Scale (Y-BOCS). Patient outcome was assessed with YBOCS and Clinical Global Impressions (CGI) scale scores at 12 weeks follow up. The results of our study and their significance are discussed.

We evaluated 105 patients, out of whom 26 patients had $\mathrm{OCD} / \mathrm{S}$. Thus the prevalence of $\mathrm{OCD} / \mathrm{Sc}$ in our study was $24.7 \%$.

\section{Demographic Variables}

All the patients in both the groups were in the age range of $19-58$ years, with nearly $51.4 \%$ patients being in the age range of $19-29$ years, $21.9 \%$ in the age range of $30-39$ years and $40-49$ years, respectively and with only $4.7 \%$ being more than 50 years. Overall male: female ratio was 1.5:1. It was seen that $50-60 \%$ of patients had achieved some form of education and nearly more than $50 \%$ patients had achieved a secondary and higher levels of education. The percentage of uneducated patients was slightly higher $(23.1 \%)$ in patients of schizophrenia with OCD as compared to patients without OCD $(11.4 \%)$. The marriage rate was higher $(76.9 \%)$ in pa-

tients of schizophrenia with OCD compared to in patients with only schizophrenia $(49.4 \%)$ and with a higher prevalence of divorced/ widowed patients(11.3\%). In our study in the OCD-schizophrenia group, most of the patients never worked $(61.5 \%), 7.7 \%$ were working and $30.8 \%$ were currently unemployed; whereas in the only schizophrenia group, $54.4 \%$ never worked, $10.5 \%$ were working and $33.3 \%$ patients were currently unemployed. In our sample, $84.6 \%$ of patients with OCD-schizophrenia and $74.7 \%$ patients with schizophrenia were staying in towns and cities with about $15-25 \%$ staying in villages. Nearly $90 \%$ of our patients belonged to the low socioeconomic status, in both the groups.

\section{Clinical Findings}

The mean duration of symptoms was 33.69 months in the OC-schizophrenia group and 24.34 months in patients with only schizophrenia. Thus the patients with OC symptoms had a significantly longer duration of schizophrenia symptoms ( $p=0.041$ ). Out of 26 patients with obsessive-compulsive disorder, the obsessive-compulsive symptoms started with schizophrenia symptoms in four (15.3\%) patients, before the onset of schizophrenia symptoms in six $(23.07 \%)$ patients and after schizophrenia symptoms in $16(61.5 \%)$ patients. The median duration of obsessive-compulsive symptoms was 19 months (range 2- 160 months).

\section{Types of OCD as per ICD 10 Criteria}

\section{Table 1: Obsessive compulsive disorder}

\begin{tabular}{lc}
$\begin{array}{l}\text { Obsessive compulsive disorder as per } \\
\text { ICD 10 Criteria }\end{array}$ & $\begin{array}{c}\text { No. of patients } \\
(\mathbf{n = 2 6})\end{array}$ \\
$\begin{array}{l}\text { Predominantly obsessional thoughts } \\
\text { and ruminations }\end{array}$ & $04(15.4 \%)$ \\
Predominantly compulsive acts & $03(11.5 \%)$ \\
Mixed obsessional thoughts and acts & $19(73.1 \%)$ \\
Other obsessive-compulsive disorders & $\mathrm{o} \mathrm{(o \% )}$ \\
Obsessive-compulsive disorder, un- & $\mathrm{o}(\mathrm{o} \%)$ \\
specified & \\
Total & $26(100 \%)$ \\
\hline
\end{tabular}

As per the ICD 10 criteria, predominantly obsessional thoughts/ruminations are diagnosed when the patient has only obsessions, predominantly compulsive acts when only ritualistic behaviors are present and mixed obsessional thoughts and act when both obsessions and compulsive acts are present.

$73.1 \%$ of our patients with OCD, (19 out of 26) fulfilled criteria for mixed obsessional thoughts and acts, where the majority had obsessional thoughts of contamination leading to compulsive washing and cleaning and checking while others had repeated obsessional thoughts of symmetry leading to repeated organizing of all the kitchen utensils in a specific 
order or thoughts of having committed a sin with compulsive acts of praying and chanting God's name thoughts of having ill health with compulsive checking of health parameters. One patient had an obsession of saving money associated with repeated checking his lockers.

$15.4 \%$ patients i.e. 4 out of 26 met criteria for predominantly obsessional thoughts. These patients had repeated blasphemous thoughts and thoughts of sexual acts with children.

$11.5 \%$ ( 3 patients) had only compulsive acts of counting sets of numbers and repeating names of objects.

Few had miscellaneous obsessions and compulsions like repeated touching rituals and asking addresses to others.

\section{Table 2: Types of OCD}

\begin{tabular}{lc}
$\begin{array}{l}\text { Type of OCD } \\
\text { as per ICD } 10\end{array}$ & $\begin{array}{c}\text { Total no. of patients } \\
(\mathbf{n = 2 6})\end{array}$ \\
\hline Mixed OCD & $19(73.0 \%)$ \\
Predominantly obsessional & $4(15.3 \%)$ \\
Predominantly compulsive & $3(11.5 \%)$ \\
Total & $26(100 \%)$ \\
\hline
\end{tabular}

Table 3: Types of Obsessions as per YBOCS

\begin{tabular}{lc} 
Obsession type & $\begin{array}{c}\text { No. of patients } \\
(\mathbf{n = 2 6})\end{array}$ \\
Aggressive Obsessions & o (o\%) \\
Contamination Obsessions & $14(53.8 \%)$ \\
Sexual Obsessions & $04(15.4 \%)$ \\
Hoarding / Saving Obsessions & $01(3.8 \%)$ \\
Religious Obsessions & $03(11.5 \%)$ \\
Obsessions with Need For Symmetry or & $01(3.8 \%)$ \\
Exactness & $01(3.8 \%)$ \\
Somatic Obsessions & $02(7.7 \%)$ \\
Miscellaneous Obsessions & $26(100 \%)$ \\
\hline
\end{tabular}

When patients of schizophrenia were diagnosed with OCD as per ICD 10 criteria were further rated on YBOCS for the type of obsessions and compulsions then we found that $53.8 \%$ (14 patients) had the obsession of contamination, $15.4 \%$ had a sexual obsession, $11.5 \%$ had a religious obsession, $7.7 \%$ had the obsession of saving, and $3.8 \%$ patients had the obsession of need for symmetry and miscellaneous obsessions each.
Table 4: Types of compulsions as per YBOCS

\begin{tabular}{lc} 
Type of Compulsion & $\begin{array}{c}\text { No. of patients } \\
(\mathbf{n}=\mathbf{2 6})\end{array}$ \\
\hline Cleaning/Washing & $14(53.8 \%)$ \\
Checking & $04(15.4 \%)$ \\
Repeating & $04(15.4 \%)$ \\
Counting & $02(7.7 \%)$ \\
Ordering/Arranging & o1 (3.8\%) \\
Hoarding/ Collecting & o (o\%) \\
Miscellaneous & o1 (3.8\%) \\
Total & $26(100 \%)$ \\
\hline
\end{tabular}

On assessing patients for types of compulsion $53 \%$ of the patients had compulsion of cleaning/washing, followed by compulsive checking and repeating in $15.4 \%$ each, $7.7 \%$ had counting rituals and $3.8 \%$ had compulsive ordering and miscellaneous rituals. This was in keeping with previous researchers who have also found a higher percentage of compulsive rituals.

\begin{tabular}{|c|c|c|}
\hline Baseline YBOCS score & Mean & Standard deviation \\
\hline YBOCS Obsessions score & 12.85 & 5.28 \\
\hline YBOCS Compulsions score & 10.92 & 5.22 \\
\hline YBOCS Total score & 23.96 & 4.06 \\
\hline
\end{tabular}

The above table shows that the mean baseline total YBOCS score was $23.96 \pm 4.06$ in our study with mean YBOCS obsessions score being $12.85 \pm 5.28$ and mean YBOCS compulsion score being $10.92 \pm 5.22$.

The severity of obsessive-compulsive symptoms can be defined on the basis of the total YBOCS score as: subclinical (YBOCS score 0-7); mild (YBOCS score 8-15); moderate (YBOCS score 16-23); severe (YBOCS score 24-31); and extreme (YBOCS score 32-40) [8]. In our study, out of 26 patients having $\mathrm{OCD} / \mathrm{S}$ with schizophrenia, 17 (65.38\%) patients had severe OC symptoms and $9(34.61 \%)$ patients had moderate OC symptoms.

In our study, out of 26 patients having $\mathrm{OCD} / \mathrm{S}$ with schizophrenia, $65.38 \%$ (17 patients) had severe OC symptoms and $34.61 \%$ (9 patients) had moderate OC symptoms. 
Improvement of patients on YBOCS at the end of 3 months

\begin{tabular}{lccccc} 
Table 6: Improvement of patients in Group A on YBOCS & & & \\
Parameter & Baseline score & Score at 3 months & t value & P value & $95 \%$ confidence Interval \\
YBOCS Obsessions score & $12.85 \pm 5.28$ & $7.27 \pm 3.32$ & 12.359 & $<0.001$ & 4.648 to 6.506 \\
YBOCS Compulsions score & $10.92 \pm 5.22$ & $6.42 \pm 3.26$ & 9.29 & $<0.001$ & 3.502 to 5.498 \\
YBOCS Total score & $23.96 \pm 4.06$ & $13.73 \pm 2.45$ & 24.154 & $<0.001$ & 9.358 to 11.103 \\
\hline
\end{tabular}

When the patients of OC with schizophrenia were assessed for improvement in their OC symptoms on the YBOCS scale at the end of 3 months then a statistically significant difference was noted on the YBOCS obsession score $(t=12.359$, $\mathrm{p}<0.001)$, YBOCS compulsion score $(\mathrm{t}=9.29, \mathrm{p}<0.001)$, and total YBOCS score $(\mathrm{t}=24.154, \mathrm{p}<0.001)$ from the baseline scores

\section{DISCUSSION}

Obsessive-compulsive disorder is an important cause of psychiatric morbidity in India. Various studies have addressed this issue in the past. Our findings correlated with the previous studies done by Berman et al., Jaydeokar et al., Porto et al. and Eisen et al. who noted the prevalence of OCD in Schizophrenia to be ranging from $25-26.7 \%{ }^{9,10,11}$.

If we consider OCD, comorbid OC-schizophrenia, and schizophrenia as a spectrum of disorders, then it is possible to explain earlier onset of symptoms in OC-schizophrenia and hence longer disease duration.

Our figures are slightly higher as compared to previous studies. It has been observed that about $12.1 \%$ patients have the onset of OC symptoms before schizophrenia symptom onset or during a period in which the patient is in at risk mental state $^{12,13,14 .}$ In a study of first-episode psychosis, the onset of OC symptoms has been noted in $17.1 \%$ patients $^{15}$.

In a study of OCD, Eisen et al. observed that the most common type of thoughts and actions as per ICD 10 criteria was mixed obsessional thoughts and acts, seen in 50\% patients, followed by predominantly obsessional thoughts and ruminations in $33.3 \%$ patients and predominantly compulsive acts in $16.6 \%$ patients ${ }^{9}$. Similarly, Jaydeokar et al. observed that among patients having OCD with schizophrenia, 85.2\% of patients had mixed obsessional thoughts and acts, whereas only $14.8 \%$ patients had predominantly obsessive thoughts and ruminations and none of the patients had predominantly compulsive acts ${ }^{11}$. Thus, the observations of our study are in agreement with these two studies.

These findings were in keeping with the previous research where the obsession with contamination was one of the most common obsessions followed by sexual obsession, aggressive thoughts, and pathological doubt. Jaydeokar et al. reported the most common obsessions to be those of contamination and sexual each in $25.92 \%$ of their patients, aggressive thoughts in $22.22 \%$ of patients, pathological doubt in $18.51 \%$, somatic in $11.11 \%$ and need for symmetry in $7.4 \%$ of their patients ${ }^{11}$. Similarly, Seedat et al. observed that the most common obsession was that of contamination $(32 \%)^{16}$

A study by de haan et al. however reported aggressive thoughts as most common obsession in $42 \%$ of the patients ${ }^{17}$. Aggressive obsessions were surprisingly not seen in our patients through a higher percentage had been reported in previous studies ${ }^{11,16}$. Jaydeokar et al. reported the most common compulsions to be the need to ask or confess in $43.47 \%$, washing in $30.43 \%$, checking in $26.08 \%$, counting in $8.69 \%$, and symmetry and hoarding compulsions in $4.34 \%$ of their patients respectively ${ }^{11}$. Similarly, Seedat et al. observed that the most common compulsion was repeating rituals $(64 \%)^{16}$. A study by de Haan et al. reported the compulsion of checking in $53 \%$ of the patients ${ }^{17}$. Our findings are in keeping with studies that have reported contamination and washing as the most common OC symptoms seen in patients.

Our findings were on the higher side to several previous studies where the mean baseline severity on OCD on YBOCS was in a range of $14 \pm 6.3$ to $17.3 \pm 4.9^{17,18,19}$. A slightly higher mean total score was found by Agrawal et al. ${ }^{20}$ at $30.3 \pm 4.9$ whereas most studies have reported the mean total YBOCS score to be ranging from about 14 to 20 . Researchers like Gulcan Gulec et al. found a baseline mean YBOCS score of $16.75 \pm 11.64$, MJ Lee et al. have observed baseline YBOCS score of $14.00 \pm 6.34$, de Haan et al. found mean baseline YBOCS score of $17.7 \pm 9.1$ whereas Hemrom et al. found it to be $17.7 \pm 9.1$ respectively $8,17,18,19$.

Furthermore, it was observed that, out of 26 patients having OCD, 18(69.23\%) patients had mild OC symptoms and 8 (30.76\%) patients had moderate OC symptoms. No patient had severe OC symptoms. Our findings were on the higher side at baseline and after 3 months but there was a significant reduction of symptoms on treatment with antipsychotics.

\section{CONCLUSIONS}

We concluded that the prevalence of OCD in the population evaluated was $24.7 \%$. It affected young individuals with 
lower socioeconomic status. Majority of patients in Group A did not work and were unemployed. The majority of patients in both groups belonged to lower socioeconomic strata. The majority of patients had mixed obsessional thought and compulsive acts pattern of OCD, followed by obsessional thoughts predominantly compulsive acts. The most common obsessions were obsessions of contamination, sexual obsession, religious obsession, savings, need for symmetry. At the end of 3 months, there was a marked reduction in obsessivecompulsive symptoms on YBOCS.

Limitations: There was a selection bias in the recruitment of the cases as the sample belonged to a tertiary care centre $\&$ does not reflect the prevalence in the general population. Comparatively small sample size. Patients were followed up for a shorter duration of time. A longer duration of follow up would give a better understanding of the $\mathrm{OC}$ symptom recurrence, prognosis, and response to treatment. The study population included only adults \& hence could not establish findings in children $\&$ adolescent population.

\section{Acknowledgement: None}

\section{Conflict of interest: None}

Financial support: None

\section{REFERENCES}

1. Hwang MY, Kim SW, Yum SY, Opler LA. Management of schizophrenia with obsessive-compulsive features. Psychiatr Clin North Am. 2009 Dec;32(4):835-51.

2. Karno M, Golding JM, Sorenson SB, et al. The epidemiology of obsessive compulsive disorder in five U.S. communities. Arch Gen Psychiatry 1988;45:1094-9.

3. Berman I, Kalinowski A, Berman SM, et al. Obsessive and compulsive symptomsin chronic schizophrenia. Compr Psychiatry 1995;36:6-10.

4. Hwang MY, Morgan JE, Losonczy MF. Clinical and neuropsychological profiles ofOC schizophrenia. J Neuropsychiatry Clin Neurosci 2000;12:91-4.

5. Sevincok L, Akoglu A, Kokcu F. Suicidality in schizophrenic patients with andwithout obsessive-compulsive disorder. Schizophr Res 2007;90:198-202.

6. Guy W, editor. ECDEU Assessment Manual for Psychopharmacology. 1976. Rockville, MD, U.S. Department of Health, Education, and Welfare

7. Hemrom S, Pushpa Prasad D, Jahan M, Singh AR, Kenswar DK. Prevalence of obsessive compulsive symptoms among patients with schizophrenia. Ind Psychiatry J. 2009 Jul;18(2):77-80.
8. Seedat F, Roos JL, Pretorius HW, Karayiorgou M, Nel B. Prevalence and clinical characteristics of obsessive-compulsive disorder and obsessive compulsive symptoms in Afrikaner schizophrenia and schizoaffective disorder patients. Afr J Psychiatry (Johannesbg). 2007 Nov;10(4):219-24.

9. Eisen JL, Beer DA, Pato MT, Venditto TA, Rasmussen SA. compulsive disorder in patients with schizophrenia or schizoaffective disorder. Am J Psychiatry 1997;154:271-3.

10. Porto L, Bermanzohn P, Siris S, Pollack S, Morrisey R. A profile of obsessive compulsive symptoms in schizophrenia. Schizophr Res 1997;24:20.

11. Thara, R., and Taj, M. (2008). Obsessive- Compulsive symptoms in schizophrenia. Eastern Journal of Psychiatry, 11:39-40.

12. Hur JW, Shin NY, Jang JH et al. "Clinical and neurocognitive profiles of subjects at high risk for psychosis with and without obsessive-compulsive symptoms," Australian and New Zealand Journal of Psychiatry. 2012; 46(2): 161-169.

13. Niendam TA, Berzak J, Cannon TD, and Bearden CE, "Obsessive compulsive symptoms in the psychosis prodrome: correlates of clinical and functional outcome," Schizophrenia Research, vol. 108, no. 1-3, pp. 170-175, 2009.

14. De Haan L, Sterk B, Wouters L, and Linszen DH, "The 5- year course of obsessive-compulsive symptoms and obsessivecompulsive disorder in first-episode schizophrenia and related disorders," Schizophrenia Bulletin. 2013; 39(1): 151-160.

15. Zink M, Schirmbeck F, Rausch F, Eifler S, Elkin H, Solojenkina $\mathrm{X}$. Obsessive-compulsive symptoms in at-risk mental states for psychosis: associations with clinical impairment and cognitive function. Acta Psychiatr Scand. 2014 Sep;130(3):214-26.

16. Ndetei DM, Pizzo M, Ongecha FA, Khasakhala LI, Maru H, Mutiso V, Kokonya DA. Obsessive-compulsive (oc) symptoms in psychiatric in-patients at Mathari hospital, Kenya. Afr J Psychiatry (Johannesbg). 2008 Aug;11(3):182-6.

17. Schirmbeck F, Rausch F, Englisch S et al."Differential effects of antipsychotic agents on obsessive-compulsive symptoms in schizophrenia: a longitudinal study," Journal of Psychopharmacology. 2013; 27(4): 349-357.

18. Lee MJ, Shin YB, Sunwoo YK, Jung SH, Kim WH, Kang MH, et al. Comparative Analysis of Cognitive Function in Schizophrenia with and without Obsessive Compulsive Disorder. Psychiatry Investig. 2009 Dec;6(4):286-93.

19. Sobin C, Roos JL, Pretorius H, Lundy LS, Karayiorgou M. A comparison study of early non-psychotic deviant behavior in Afrikaner and US patients with schizophrenia or schizoaffective disorder. Psychiatry Res. 2003 Feb 15;117(2):113-25.

20. Güleç G, Güneş E, Yenilmez C. Comparison of patients with schizophrenia, obsessive-compulsive disorder, and schizophrenia with accompanying obsessive-compulsive symptoms. Turk Psikiyatri Derg. 2008 Fall;19(3):247-56. 